\title{
Parity violating gravitational response and anomalous constitutive relations
}

\author{
Juan L. Mañes ${ }^{a}$ and Manuel Valle ${ }^{b}$ \\ ${ }^{a}$ Departamento de Física de la Materia Condensada, Universidad del País Vasco UPV/EHU, \\ Apartado 644, 48080 Bilbao, Spain \\ ${ }^{b}$ Departamento de Física Teórica, Universidad del País Vasco UPV/EHU, \\ Apartado 644, 48080 Bilbao, Spain \\ E-mail: wmpmapaj@lg.ehu.es, manuel.valle@ehu.es
}

\begin{abstract}
We compute the parity violating part of the time-dependent gravitational response function of an ideal gas of Weyl fermions up to third order in the derivative expansion and give its full tensorial structure. Our main results are two functions that parametrize the energy-momentum tensor in terms of gauge-invariant combinations of vector and tensor metric perturbations. The zero frequency limit of these functions is related with the anomalous constitutive relations and with the full anomalous partition function in the presence of gauge and mixed anomalies. In particular, our results imply the existence of a previously unknown invariant contribution to the parity-odd partition function at third derivative order that we explicitly construct. Beyond the static limit, the gravitational response function may provide valuable insights into time-dependent phenomena driven by anomalies.
\end{abstract}




\section{Contents}

1 Introduction $\quad 1$

2 Leading contribution at low momentum 3

3 Parity-odd response function $\quad 4$

3.1 Leading contribution to the parity-odd response function 5

3.2 Parity-odd response function at higher orders in the momenta 8

4 Energy-momentum tensor and metric perturbations $\quad 9$

4.1 Static limit of response functions and anomalous constitutive relations $\quad 10$

5 The anomalous partition function and the static response function $\quad \mathbf{1 3}$

$\begin{array}{ll}5.1 \text { The first order generating functional } & 13\end{array}$

$\begin{array}{ll}5.2 \text { The third order generating functional } & 14\end{array}$

$\begin{array}{llr}6 & \text { Discussion and outlook } & 18\end{array}$

$\begin{array}{ll}\text { A Expansion of the action and seagull terms } & 18\end{array}$

$\begin{array}{ll}\text { B The integrals } I^{\mu \nu \rho} & 19\end{array}$

$\begin{array}{ll}\text { C The integrals } I^{\mu \nu} & 21\end{array}$

\section{Introduction}

One of the most interesting and surprising developments in thermal field theory has been the recent realization that anomalies induce unexpected modifications in the constitutive relations of relativistic hydrodynamics [1-3]. As a consequence, there are allowed transport phenomena [4-6] related only to anomalies which manifest themselves by parity violation. An important feature of the new anomalous terms is that they do not break time-reversal symmetry and hence the equilibrium response to a perturbation is not accompanied by an increase in entropy. In this regard, it has been argued that a systematic derivation of constraints on the constitutive relations should be possible solely from the knowledge of a consistent functional describing the equilibrium thermodynamics, without having to make use of an entropy current [7-9]. More specifically, this functional has been constructed for an anomalous charged fluid, to second order in the derivative expansion in $1+1$ dimensions [10], and to third order in $3+1$ dimensions [11]. These constructions, supplemented by the appropriate Bardeen-Zumino terms [11-13] to be added to the consistent currents, provide higher-order anomalous contributions to the constitutive relations due to gravitational and mixed anomalies. 
As mentioned above, an important feature of the anomalous contributions to the constitutive relations is that they break invariance under parity. To see the implications of this fact, let us consider the non-relativistic limit of the anomalous constitutive relation connecting the vorticity to the momentum density $[14,15]$

$$
\boldsymbol{g}=\chi_{P V} \nabla \times \boldsymbol{v}
$$

where $\boldsymbol{v}$ is the fluid velocity in a generic frame (not in the Landau frame). According to the theory of hydrodynamic fluctuations [16], the non-zero static susceptibility $\chi_{P V}$ is given by the following sum rule

$$
\chi_{P V} \propto \lim _{q \rightarrow 0} \frac{i q^{j} \epsilon^{j m n}}{q^{2}} \int_{-\infty}^{\infty} d \omega \frac{\chi_{g g}^{m n}(\omega, \boldsymbol{q})}{\omega},
$$

in terms of the imaginary part $\chi_{g g}^{i j}(\omega, \boldsymbol{q})$ of the retarded momentum-momentum correlator. For a translationally invariant system this correlator takes the general form

$$
\chi_{g g}^{i j}(\omega, \boldsymbol{q})=\frac{q^{i} q^{j}}{q^{2}} \chi_{L}(\omega, q)+\left(\delta^{i j}-\frac{q^{i} q^{j}}{q^{2}}\right) \chi_{T}(\omega, q)+i \epsilon^{i j k} q^{k} \chi_{P V}(\omega, q) .
$$

Since all the momentum density components $g^{i}$ have the same signature under discrete symmetries, the last term of (1.3) can be different from zero only if the equilibrium state used in the definition of $\chi_{g g}^{i j}(t, \boldsymbol{x}, 0, \mathbf{0})=\left\langle\left[g^{i}(t, \boldsymbol{x}), g^{j}(0, \mathbf{0})\right]\right\rangle$ is not invariant under parity. For a system of chiral fermions this requires a nonzero axial chemical potential, and $\chi_{P V}$ is expected to be an odd function of this potential. This observation suggests that the study of the parity violating piece of the two-point function for gravitons may be useful in order to deepen our understanding of anomalies in hydrodynamics and also to gain information about time-dependent phenomena driven by anomalies, a field that remains relatively unexplored.

In this paper we undertake the computation of the parity violating part of the gravitational response function of an ideal gas of Weyl fermions at small frequencies and momenta compared to the chemical potential and temperature. In other words, we compute the gravitational response function in the hard-dense-loop approximation. The procedure is based on previous work by Rebhan [17], who has given the full tensorial structure of the leading corrections $\propto T^{4}$ of the graviton self-energy. A brief review of the work by Rebhan, generalized to non-vanishing chemical potential, is presented in the next section, where the resulting energy-momentum tensor is shown to correspond to the constitutive relations of a perfect conformal fluid at lowest order in the derivative expansion of the fluid fields. This is extended to third order in the derivative expansion in section 3 , where our main results are two functions that parametrize the energy-momentum tensor in terms of gauge-invariant combinations of vector and tensor metric perturbations. The zero frequency limit of these functions is related with the anomalous constitutive relations in section 4, and with the form of the full anomalous partition function in the presence of gauge and mixed anomalies in section 5, where we find a previously unknown invariant contribution to the parity-odd partition function. Our conclusions and possible applications of this work to the study of time-dependent hydrodynamic phenomena driven by anomalies are presented in section 6 . 


\section{Leading contribution at low momentum}

In this section we briefly review previous work by Rebhan [17] on the graviton self-energy at leading order, and connect it with the constitutive relations of a perfect conformal fluid. The graviton polarization tensor is defined by

$$
\Pi^{\mu \nu \rho \sigma}(x-y)=-\left.4 \frac{\delta \Gamma}{\delta g_{\mu \nu}(x) \delta g_{\rho \sigma}(y)}\right|_{g=\eta}=-\left.2 \frac{\delta}{\delta g_{\mu \nu}(x)}\left(\sqrt{-g}\left\langle T^{\rho \sigma}(y)\right\rangle\right)\right|_{g=\eta},
$$

where $\Gamma$ is the effective action and

$$
\left\langle T^{\mu \nu}\right\rangle=\frac{2}{\sqrt{-g}} \frac{\delta \Gamma}{\delta g_{\mu \nu}}
$$

Note that eq. (2.1) implies

$$
\delta\left(\sqrt{-g}\left\langle T^{\mu \nu}(x)\right\rangle\right)=-\frac{1}{2} \int d^{4} y \Pi^{\mu \nu \rho \sigma}(x-y) h_{\rho \sigma}(y),
$$

where the retarded version of $\Pi^{\mu \nu \rho \sigma}(x-y)$ has to be used in order to compute the corresponding induced change to linear order in $h_{\mu \nu} \equiv g_{\mu \nu}-\eta_{\mu \nu}$.

It was shown in [17] that, for small momenta $\left|q^{0}\right|, q \ll T_{0}, \mu_{0}$ the leading behavior of $\Pi^{\mu \nu \rho \sigma}(Q)$ can be written in terms of the integral

$$
I^{\mu \nu \rho \sigma}(Q)=T_{0} \sum_{\omega_{m}} \int \frac{d^{3} k}{(2 \pi)^{3}} \frac{K^{\mu} K^{\nu} K^{\rho} K^{\sigma}}{K^{2}(K+Q)^{2}}, \quad K^{\mu}=\left(i \omega_{m}+\mu_{0}, \boldsymbol{k}\right), \quad Q^{\mu}=\left(i \nu_{n}, \boldsymbol{q}\right),
$$

where $\nu_{n}\left(\omega_{n}\right)$ are bosonic (fermionic) Matsubara frequencies, and the pure vacuum $\left(T_{0}=\right.$ $\left.\mu_{0}=0\right)$ divergence has been subtracted. Concretely, the following combination of indices,

$$
\Pi^{\mu \nu \rho \sigma}(Q)=2 I^{\mu \nu \rho \sigma}(Q)-\eta_{\alpha \beta}\left(I^{\alpha \beta \mu \rho} \eta^{\nu \sigma}+I^{\alpha \beta \nu \rho} \eta^{\mu \sigma}+I^{\alpha \beta \mu \sigma} \eta^{\nu \rho}+I^{\alpha \beta \nu \sigma} \eta^{\mu \rho}\right),
$$

produces the correct graviton polarization tensor satisfying the Ward identities implied by general covariance. After the sum over Matsubara frequencies is performed, the small momentum behavior of $I^{\mu \nu \lambda \sigma}(Q)$ can be extracted by making the rescaling $q^{0}=i \nu_{n} \rightarrow$ $\epsilon q^{0}, \boldsymbol{q} \rightarrow \epsilon \boldsymbol{q}$, and keeping only $\mathcal{O}\left(\epsilon^{0}\right)$ terms. The integrand obtained in this way is proportional to the energy density which, for a left (or right)-handed Weyl field, is given by

$$
\varepsilon=\int_{0}^{\infty} \frac{d k k^{2}}{2 \pi^{2}} k\left(n_{F}\left(k-\mu_{0}\right)+n_{F}\left(k+\mu_{0}\right)\right)=\frac{7 \pi^{2} T_{0}^{4}}{120}+\frac{\mu_{0}^{2} T_{0}^{2}}{4}+\frac{\mu_{0}^{4}}{8 \pi^{2}} .
$$

The remaining angular integration yields the entire dependence of $I^{\mu \nu \lambda \sigma}$ on $q^{0} /|\boldsymbol{q}|$. In particular, the explicit computation of $I^{\mu \nu \rho \sigma}(Q)$ in the static limit $\left(q^{0}=0\right)$ yields the following non-vanishing components [17] for the polarization tensor

$$
\begin{aligned}
& \Pi^{0000}=-5 \varepsilon, \quad \Pi^{00 i i}=-\varepsilon, \quad \Pi^{0 i 0 i}=-\frac{\varepsilon}{3}, \\
& \Pi^{i i j j}=-\frac{\varepsilon}{3}, \quad \quad \Pi^{i i 00}=-\varepsilon, \quad \Pi^{i j i j}=\frac{\varepsilon}{3},
\end{aligned}
$$


where the value of $\Pi^{i i j j}$ is valid only for $i \neq j$, with $\Pi^{i i i i}=\varepsilon / 12$.

An important feature of these results is that its form exactly corresponds to the constitutive relation of a perfect conformal fluid at lowest order in the derivative expansion of the fluid fields

$$
\left\langle T^{\mu \nu}\right\rangle=(\varepsilon+P) u^{\mu} u^{\nu}+P g^{\mu \nu} .
$$

This can be seen by considering the system in a curved background. The most general static metric which is preserved by the Killing vector $\partial_{t}$ may be written as [9]

$$
d s^{2}=-e^{2 \sigma(\vec{x})}\left(d t+a_{j}(\vec{x}) d x^{j}\right)^{2}+g_{i j}(\vec{x}) d x^{i} d x^{j} .
$$

Now, in a comoving coordinate system, the fluid velocity becomes $u^{\mu}=\delta_{0}^{\mu} e^{-\sigma}$, and the temperature and chemical potential also acquire a dependence on the position given by [18]

$$
\begin{aligned}
T(\vec{x}) & =\left(-g_{00}\right)^{-1 / 2} T_{0}=e^{-\sigma} T_{0}, \\
\mu(\vec{x}) & =e^{-\sigma} \mu_{0} .
\end{aligned}
$$

Here $T_{0}$ and $\mu_{0}$ are constants which may be viewed as the temperature and chemical potential in the absence of gravity ${ }^{1}$. Thus, in the conformal case $P=\varepsilon / 3$, the induced corrections to $\left\langle T^{\mu \nu}\right\rangle$ are simply obtained by making the replacements $\varepsilon \rightarrow e^{-4 \sigma} \varepsilon, u^{\nu} \rightarrow$ $e^{-\sigma} \delta_{0}^{\nu}$ and $\eta^{\mu \nu} \rightarrow g^{\mu \nu}$. To linear order in $h_{\mu \nu}$ this yields

$$
\begin{aligned}
\delta\left(\sqrt{-g}\left\langle T^{00}\right\rangle\right) & =\frac{5 \varepsilon}{2} h_{00}+\frac{\varepsilon}{2} \sum_{k} h_{k k}, \\
\delta\left(\sqrt{-g}\left\langle T^{0 i}\right\rangle\right) & =\frac{\varepsilon}{3} h_{0 i}, \\
\delta\left(\sqrt{-g}\left\langle T^{i i}\right\rangle\right) & =\frac{\varepsilon}{2} h_{00}-\frac{\varepsilon}{3} h_{i i}+\frac{\varepsilon}{6} \sum_{k} h_{k k}, \\
\delta\left(\sqrt{-g}\left\langle T^{i j}\right\rangle\right) & =-\frac{\varepsilon}{3} h_{i j}, \quad i \neq j,
\end{aligned}
$$

where $h_{00}=-2 \sigma, h_{0 k}=-a_{k}$ and $h_{i j}=g_{i j}-\delta_{i j}$. A look at (2.7) shows that the coefficients in (2.11) exactly agree with the components of $\Pi^{\mu \nu \rho \sigma}$ in the static limit. Thus, they encode the form of the constitutive relations for the energy-momentum tensor at the lowest order in a derivative expansion of the fluid fields. In the remaining of this paper we will extend Rebhan's computation to higher orders in the momenta and analyze the implications for the constitutive relations and parity violating partition function.

\section{Parity-odd response function}

As explained in the Introduction, our interest will be in the parity violating part of the gravitational response function. From the fact that $\left\langle T^{\mu \nu}\right\rangle$ in flat space-time is of the form (2.8), which is parity-even, it follows that, in order to obtain the parity-odd part of $\delta\left\langle T^{\mu \nu}(x)\right\rangle$, eq. (2.3) can be simplified to

$$
\delta\left\langle T^{\mu \nu}(x)\right\rangle=-\frac{1}{2} \int d^{4} y \Pi^{\mu \nu \rho \sigma}(x-y) h_{\rho \sigma}(y),
$$

\footnotetext{
${ }^{1}$ Note that $T_{0}$ and $\mu_{0}$ are the same constants that appear in the partition function $Z=\operatorname{Tr} e^{-\left(H-\mu_{0} N\right) / T_{0}}$ even if the Hamiltonian includes the static gravitational field.
} 
where the required response function is given by

$$
\Pi^{\mu \nu \rho \sigma}(x-y) \equiv-i \theta\left(x^{0}-y^{0}\right)\left\langle\left[T^{\mu \nu}(x), T^{\rho \sigma}(y)\right]\right\rangle-2\left\langle\left.\frac{\delta\left(\sqrt{-g(x)} T^{\mu \nu}(x)\right)}{\delta g_{\rho \sigma}(y)}\right|_{g=\eta}\right\rangle .
$$

For an ideal gas of left-handed Weyl fermions, the first term in (3.2) takes the following form in the imaginary time formalism

$$
\begin{aligned}
\Pi_{1}^{\mu \nu \rho \sigma}\left(i \nu_{n}, \boldsymbol{q}\right)= & T \sum_{\omega_{n}} \int \frac{d^{3} k}{(2 \pi)^{3}} \operatorname{tr}\left[\mathcal{P}_{-} \not K V^{\mu \nu}(K, K+Q)\left(K_{K}+\not\right)\right. \\
& \left.\times V^{\rho \sigma}(K+Q, K)\right] \frac{1}{K^{2}(K+Q)^{2}}, \quad K^{0}=i \omega_{n}+\mu,
\end{aligned}
$$

where $\mathcal{P}_{-}=\left(1-\gamma_{5}\right) / 2$ and the fermion-fermion-graviton three-vertex, which can be read from the energy-momentum tensor $T^{\mu \nu}$ in Minkowski space (A.3), is given by ${ }^{2}$

$$
V^{\mu \nu}(K, P)=\frac{1}{4}\left[\gamma^{\mu}(K+P)^{\nu}+\gamma^{\nu}(K+P)^{\mu}\right]-\frac{1}{2} \eta^{\mu \nu}(K K+\not P) .
$$

Up to parity even contributions (see appendix A for details) the second term in (3.2), coming from the seagull diagram, can be written

$$
\begin{aligned}
\Pi_{2}^{\mu \nu \rho \sigma}\left(i \nu_{n}, \boldsymbol{q}\right)= & \frac{1}{8} \eta^{\mu \rho} T \sum_{\omega_{n}} \int \frac{d^{3} k}{(2 \pi)^{3}} \operatorname{tr}\left[\left\{\sigma^{\nu \sigma}, \not \ell\right\} \mathcal{P}_{-} \not K\right] \frac{1}{K^{2}} \\
& +\frac{1}{8} \eta^{\nu \rho} T \sum_{\omega_{n}} \int \frac{d^{3} k}{(2 \pi)^{3}} \operatorname{tr}\left[\left\{\sigma^{\mu \sigma}, \not \subset\right\} \mathcal{P}_{-} \not K\right] \frac{1}{K^{2}}+(\rho \leftrightarrow \sigma),
\end{aligned}
$$

where $\sigma^{\nu \sigma} \equiv \frac{1}{4}\left[\gamma^{\nu}, \gamma^{\sigma}\right]$. Here the zero subscript from $T$ and $\mu$ has been omitted.

\subsection{Leading contribution to the parity-odd response function}

As reviewed in the previous section, for momenta $\left|q^{0}\right|, q \ll|\mu|, T$, the leading behavior of $\Pi^{\mu \nu \rho \sigma}$ is proportional to the energy density and can be written in terms the prototype integral (2.4). The next to leading order contribution to $\Pi^{\mu \nu \rho \sigma}$ in the momentum expansion, which is odd in the chemical potential and linear in $Q$, turns out to be governed by the integral

$$
I^{\mu \nu \lambda}(Q)=T \sum_{\omega_{n}} \int \frac{d^{3} k}{(2 \pi)^{3}} \frac{K^{\mu} K^{\nu} K^{\lambda}}{K^{2}(K+Q)^{2}} .
$$

Concretely, the leading parity-odd contribution to $\Pi_{1}$ is obtained by picking the $\gamma_{5}$ in the projector $\mathcal{P}_{-}=\left(1-\gamma_{5}\right) / 2$ together with as many $K$ s as possible in the numerator of (3.3). This gives

$$
\Pi_{1}^{\mu \nu \rho \sigma}\left(q^{0}, \boldsymbol{q}\right)=\frac{i}{2} Q_{\alpha} \eta_{\beta \lambda}\left(\epsilon^{\alpha \beta \mu \rho} I^{\nu \sigma \lambda}+\epsilon^{\alpha \beta \nu \rho} I^{\mu \sigma \lambda}\right)+(\rho \leftrightarrow \sigma) .
$$

\footnotetext{
${ }^{2}$ The gamma matrices obey $\left\{\gamma^{\mu}, \gamma^{\nu}\right\}=-2 \eta^{\mu \nu}, \gamma_{5}=-\frac{i}{24} \epsilon_{\mu \nu \rho \sigma} \gamma^{\mu} \gamma^{\nu} \gamma^{\rho} \gamma^{\sigma}$, where $\epsilon_{0123}=-\epsilon^{0123}=-1$. Note that we use the mostly positive signature.
} 
Similarly, the parity-odd part of $\Pi_{2}$ is given by

$$
\Pi_{2}^{\mu \nu \rho \sigma}\left(q^{0}, \boldsymbol{q}\right)=-\frac{i}{4} Q_{\alpha} \eta_{\beta \lambda}\left(\epsilon^{\alpha \beta \mu \rho} \eta^{\nu \sigma} H^{\lambda}+\epsilon^{\alpha \beta \nu \rho} \eta^{\mu \sigma} H^{\lambda}\right)+(\rho \leftrightarrow \sigma),
$$

where

$$
H^{\mu}=T \sum_{\omega_{n}} \int \frac{d^{3} k}{(2 \pi)^{3}} \frac{K^{\mu}}{K^{2}}
$$

As before, the evaluation of (3.6) involves Matsubara summation and rescaling of the resulting integrand through $q^{0} \rightarrow \epsilon q^{0}, \boldsymbol{q} \rightarrow \epsilon \boldsymbol{q}$. The integral obtained by keeping the $\mathcal{O}\left(\epsilon^{0}\right)$ term, which we will denote $I_{(0)}^{\mu \nu \lambda}$, has all components proportional to

$$
I_{(0)}=\int_{0}^{\infty} \frac{d k}{4 \pi^{2}} k^{2}\left(n_{F}(k-\mu)-n_{F}(k+\mu)\right)=\frac{1}{12 \pi^{2}}\left(\mu^{3}+\pi^{2} \mu T^{2}\right) .
$$

In particular, we have

$$
\eta_{\alpha \beta} I_{(0)}^{\alpha \beta \gamma}=H^{\gamma}=-I_{(0)} u^{\gamma},
$$

where $u^{\gamma}=(1, \mathbf{0})$ is the velocity of the fluid in the local rest frame. Using this relation to eliminate $H^{\lambda}$ in favor of $I_{(0)}^{\mu \nu \lambda}$ and adding the contributions of $\Pi_{1}$ and $\Pi_{2}$, gives the following expression for the leading parity-odd contribution to the polarization tensor

$$
\begin{aligned}
\Pi_{(1)}^{\mu \nu \rho \sigma}\left(q^{0}, \boldsymbol{q}\right)= & \frac{i}{2} Q_{\alpha} \eta_{\beta \lambda} \epsilon^{\alpha \beta \mu \rho}\left(I_{(0)}^{\nu \sigma \lambda}-\frac{1}{2} \eta^{\nu \sigma} \eta_{\gamma \kappa} I_{(0)}^{\gamma \kappa \lambda}\right)+\ldots \\
= & \frac{i}{4} Q_{\alpha} \eta_{\beta \lambda} I_{(0)}^{\gamma \kappa \lambda}\left[\epsilon^{\alpha \beta \mu \rho}\left(\delta_{\gamma \kappa}^{\nu \sigma}-\eta^{\nu \sigma} \eta_{\gamma \kappa}\right)\right. \\
& \left.+\epsilon^{\alpha \beta \nu \rho}\left(\delta_{\gamma \kappa}^{\mu \sigma}-\eta^{\mu \sigma} \eta_{\gamma \kappa}\right)+(\rho \leftrightarrow \sigma)\right],
\end{aligned}
$$

where $\delta_{\gamma \kappa}^{\nu \sigma}=\delta_{\gamma}^{\nu} \delta_{\kappa}^{\sigma}+\delta_{\gamma}^{\sigma} \delta_{\kappa}^{\nu}$.

We can check this result by noting that the covariant conservation law for the energymomentum tensor imposes constraints on the tensorial structure of $\Pi^{\mu \nu \rho \sigma}(Q)$. Since the covariant derivative involves a combination of derivatives of $\delta\left\langle T^{\mu \nu}\right\rangle$ and Christoffel symbols, and both of them are of the same order in $h_{\rho \sigma}$, it follows that the vanishing of $\nabla_{\mu} \delta\left\langle T^{\mu \nu}(x)\right\rangle$ gives rise to Ward identities connecting $Q_{\mu} \Pi^{\mu \nu \rho \sigma}(Q)$ with the one-point function $\left\langle T^{\alpha \beta}\right\rangle[19]$. The consequences of this requirement in relation with (2.4) were fully analyzed in [17]. With regard to the parity-violating part of $\Pi^{\mu \nu \rho \sigma}(Q)$, the conservation law imposes uniquely $Q_{\mu} \Pi^{\mu \nu \rho \sigma}(Q)=0$, since the parity-violating part of $\left\langle T^{\alpha \beta}\right\rangle$ vanishes. Similarly, conformal invariance requires $\eta_{\mu \nu} \Pi^{\mu \nu \rho \sigma}(Q)=0$ for this part. Now, $\Pi_{(1)}^{\mu \nu \rho \sigma}$ as given by (3.12) is obviously traceless, while transversality follows from the following property satisfied by the componets of $I^{\mu \nu \lambda}$ at leading order

$$
I_{(0)}^{\mu \nu \lambda} Q_{\lambda}=\frac{1}{2}\left(Q^{\mu} \delta_{\gamma}^{\nu}+Q^{\nu} \delta_{\gamma}^{\mu}\right) \eta_{\alpha \beta} I_{(0)}^{\alpha \beta \gamma}
$$

which is easily proved from the results in table 2 of appendix B. Actually, up to an overall normalization, the structure of the leading parity-odd response function (3.12) is uniquely determined by the conditions of transversality and tracelessness together with (3.13). 
In order to evaluate $\Pi_{(1)}^{\mu \nu \rho \sigma}$ we need explicit expressions for all the components of $I^{\mu \nu \lambda}$. This is conveniently done by using the decomposition $I^{\mu \nu \lambda}=\sum_{j} c_{j} T_{j}^{\mu \nu \lambda}$ in terms of the six symmetric tensors

$$
\begin{aligned}
& T_{1}^{\mu \nu \lambda}=Q^{\mu} Q^{\nu} Q^{\lambda}, \\
& T_{2}^{\mu \nu \lambda}=Q^{\mu} Q^{\nu} u^{\lambda}+Q^{\mu} u^{\nu} Q^{\lambda}+u^{\mu} Q^{\nu} Q^{\lambda}, \\
& T_{3}^{\mu \nu \lambda}=Q^{\mu} u^{\nu} u^{\lambda}+u^{\mu} Q^{\nu} u^{\lambda}+u^{\mu} u^{\nu} Q^{\lambda}, \\
& T_{4}^{\mu \nu \lambda}=Q^{\mu} \eta^{\nu \lambda}+Q^{\nu} \eta^{\mu \lambda}+Q^{\lambda} \eta^{\mu \nu}, \\
& T_{5}^{\mu \nu \lambda}=u^{\mu} u^{\nu} u^{\lambda}, \\
& T_{6}^{\mu \nu \lambda}=u^{\mu} \eta^{\nu \lambda}+u^{\nu} \eta^{\mu \lambda}+u^{\lambda} \eta^{\mu \nu} .
\end{aligned}
$$

The contractions of $I^{\mu \nu \lambda}$ with the basis tensors are given in table 2 of appendix B. Inverting these relations gives the coefficients $c_{j}$

$$
\begin{aligned}
& c_{1}\left(q^{0}, q\right) / I_{(0)}=-\frac{5 q^{0}}{4 q^{4}}-\frac{3 q^{0}\left(5 Q^{2}-2 q^{2}\right)}{4 q^{6}} L\left(q^{0}, q\right), \\
& c_{2}\left(q^{0}, q\right) / I_{(0)}=-\frac{5 Q^{2}-2 q^{2}}{4 q^{4}}-\frac{3 Q^{2}\left(5 Q^{2}-4 q^{2}\right)}{4 q^{6}} L\left(q^{0}, q\right), \\
& c_{3}\left(q^{0}, q\right) / I_{(0)}=\frac{q^{0}\left(5 Q^{2}+2 q^{2}\right)}{4 q^{4}}+\frac{15 q^{0} Q^{4}}{4 q^{6}} L\left(q^{0}, q\right), \\
& c_{4}\left(q^{0}, q\right) / I_{(0)}=\frac{q^{0}}{4 q^{2}}+\frac{3 q^{0} Q^{2}}{4 q^{4}} L\left(q^{0}, q\right), \\
& c_{5}\left(q^{0}, q\right) / I_{(0)}=Q^{2}\left(\frac{5 Q^{2}+2 q^{2}}{4 q^{4}}+\frac{15 Q^{4}}{4 q^{6}} L\left(q^{0}, q\right)\right), \\
& c_{6}\left(q^{0}, q\right) / I_{(0)}=Q^{2}\left(\frac{1}{4 q^{2}}+\frac{3 Q^{2}}{4 q^{4}} L\left(q^{0}, q\right)\right),
\end{aligned}
$$

where $L\left(q^{0}, q\right) \equiv Q_{1}\left(q^{0} / q\right)$ is the Legendre function of the second kind, which results from using the retarded prescription $i \nu_{n}+0^{+} \rightarrow q^{0}$ in the analytic continuation of the integral

$$
\int_{-1}^{1} d t \frac{t}{i \nu_{n}-q t} \rightarrow \frac{2}{q} L\left(q^{0}, q\right)=\frac{2}{q}\left[-1+\frac{q^{0}}{2 q} \ln \left|\frac{q^{0}+q}{q^{0}-q}\right|-\frac{i \pi}{2} \frac{q^{0}}{q} \theta\left(1-\frac{\left(q^{0}\right)^{2}}{q^{2}}\right)\right] .
$$

This result can be greatly simplified by using the constraints imposed by (3.13), which restrict the number of independent functions to three, for example $c_{1}, c_{2}$ and $c_{3}$. Clearly $c_{1}$ does not contribute, and we are left with only two combinations of the functions $c_{2}$ and $c_{3}$. Due to the transversality of the response function, the result can be written in a particularly transparent way in terms of the two projectors $P_{\mathbb{T}}$ and $P_{\mathbb{V}}$,

$$
\begin{aligned}
& P_{\mathbb{T}}^{\mu \nu}=\eta^{\mu \nu}-\frac{1}{(u \cdot Q)^{2}+Q^{2}}\left[u \cdot Q\left(u^{\mu} Q^{\nu}+u^{\nu} Q^{\nu}\right)+Q^{\mu} Q^{\nu}-Q^{2} u^{\mu} u^{\nu}\right], \\
& P_{\mathbb{V}}^{\mu \nu}=\eta^{\mu \nu}-\frac{Q^{\mu} Q^{\nu}}{Q^{2}}-P_{\mathbb{T}}^{\mu \nu} .
\end{aligned}
$$


Then the response function (3.12) adopts the simple form

$$
\begin{aligned}
\Pi_{(1)}^{\mu \nu \rho \sigma}\left(q^{0}, \boldsymbol{q}\right)= & i c_{\mathbb{V}}\left(q^{0}, q\right) \frac{Q^{2}}{(u \cdot Q)^{2}+Q^{2}} u_{\alpha} Q_{\beta}\left[\epsilon^{\alpha \beta \mu \rho} P_{\mathbb{V}}^{\nu \sigma}+\epsilon^{\alpha \beta \nu \rho} P_{\mathbb{V}}^{\mu \sigma}+(\rho \leftrightarrow \sigma)\right] \\
& +i c_{\mathbb{T}}\left(q^{0}, q\right) u_{\alpha} Q_{\beta}\left[\epsilon^{\alpha \beta \mu \rho} P_{\mathbb{T}}^{\nu \sigma}+\epsilon^{\alpha \beta \nu \rho} P_{\mathbb{T}}^{\mu \sigma}+(\rho \leftrightarrow \sigma)\right],
\end{aligned}
$$

where the functions $c_{\mathbb{L}, \mathbb{T}}$ are given by

$$
\begin{aligned}
c_{\mathbb{V}}\left(q^{0}, q\right) & =\frac{q^{2}}{2 q^{0}}\left(q^{0} c_{2}+c_{3}\right) \\
& =\frac{1}{24 \pi^{2}}\left(\mu^{3}+\pi^{2} \mu T^{2}\right)\left(1+\frac{3 Q^{2}}{q^{2}} L\left(q^{0}, q\right)\right), \\
c_{\mathbb{T}}\left(q^{0}, q\right) & =\frac{1}{2}\left[Q^{2} c_{2}-q^{0} c_{3}\right] \\
& =-\frac{1}{96 \pi^{2}}\left(\mu^{3}+\pi^{2} \mu T^{2}\right)\left(2+\frac{Q^{2}}{q^{2}}+\frac{3 Q^{4}}{q^{4}} L\left(q^{0}, q\right)\right) .
\end{aligned}
$$

In the static limit these become

$$
\begin{aligned}
& c_{\mathbb{V}}(0, q)=-\frac{1}{12 \pi^{2}}\left(\mu^{3}+\pi^{2} \mu T^{2}\right), \\
& c_{\mathbb{T}}(0, q)=0 .
\end{aligned}
$$

These are the main results in this subsection.

\subsection{Parity-odd response function at higher orders in the momenta}

In order to use the response function as a source of constraints on the anomalous constitutive relations at higher order in the derivative expansion, we will need $\Pi^{\mu \nu \rho \sigma}(Q)$ up to third order in $Q$. The form of the second order contribution comes from the explicit contribution of order $Q^{2}$ to the trace in (3.3) together with the piece of $\mathcal{O}(Q)$ in $I^{\alpha \beta \gamma}$. From the explicit formula (3.5) for $\Pi_{2}$, it is obvious that the seagull diagram does not contribute beyond first order. Therefore the second order correction reads

$$
\begin{aligned}
\Pi_{(2)}^{\mu \nu \rho \sigma}(Q)= & \frac{i}{2} Q_{\alpha} \eta_{\beta \lambda} \epsilon^{\alpha \beta \mu \rho}\left(I_{(1)}^{\nu \sigma \lambda}+\frac{1}{2}\left(Q^{\nu} I_{(0)}^{\sigma \lambda}+Q^{\sigma} I_{(0)}^{\nu \lambda}\right)\right) \\
& \frac{i}{2} Q_{\alpha} \eta_{\beta \lambda} \epsilon^{\alpha \beta \nu \rho}\left(I_{(1)}^{\mu \sigma \lambda}+\frac{1}{2}\left(Q^{\mu} I_{(0)}^{\sigma \lambda}+Q^{\sigma} I_{(0)}^{\mu \lambda}\right)\right)+(\rho \leftrightarrow \sigma),
\end{aligned}
$$

where we have introduced the integral

$$
I^{\alpha \beta}(Q)=T \sum_{k^{0}} \int \frac{d^{3} k}{(2 \pi)^{3}} \frac{K^{\alpha} K^{\beta}}{K^{2}(K+Q)^{2}},
$$

which in this case is needed only at $\mathcal{O}\left(Q^{0}\right)$. A computation using the results for the integrals in appendices $\mathrm{B}$ and $\mathrm{C}$ shows that, although neither $I_{(0)}^{\alpha \beta}$ nor $I_{(1)}^{\alpha \beta \gamma}$ vanish, the specific combination in (3.21) does. Therefore the second order contribution to the parity odd gravitational response function vanishes identically. 
The third order contribution may be expressed similarly. Besides $I^{\alpha \beta \gamma}$ at $\mathcal{O}\left(Q^{2}\right)$ and $I^{\alpha \beta}(Q)$ at $\mathcal{O}(Q)$, we also need the $\mathcal{O}\left(Q^{0}\right)$ piece of the integral $I^{\mu}$, which turns out to be independent of the temperature

$$
I^{\mu}=T \sum_{k^{0}} \int \frac{d^{3} k}{(2 \pi)^{3}} \frac{K^{\mu}}{K^{2}(K+Q)^{2}} \sim \frac{\mu L\left(q^{0}, q\right)}{8 \pi^{2}}\left(\frac{Q^{2}}{q^{2}} u^{\mu}+\frac{q^{0}}{q^{2}} Q^{\mu}\right) .
$$

The appropriate combination is now

$$
\begin{aligned}
\Pi_{(3)}^{\mu \nu \rho \sigma}\left(q^{0}, \boldsymbol{q}\right)= & \frac{i}{2} Q_{\alpha} \eta_{\beta \lambda} \epsilon^{\alpha \beta \mu \rho}\left(I_{(2)}^{\nu \sigma \lambda}+\frac{1}{2}\left(Q^{\nu} I_{(1)}^{\sigma \lambda}+Q^{\sigma} I_{(1)}^{\nu \lambda}\right)+\frac{1}{4} Q^{\nu} Q^{\sigma} I_{(0)}^{\lambda}\right) \\
& +\frac{i}{2} Q_{\alpha} \eta_{\beta \lambda} \epsilon^{\alpha \beta \nu \rho}\left(I_{(2)}^{\mu \sigma \lambda}+\frac{1}{2}\left(Q^{\mu} I_{(1)}^{\sigma \lambda}+Q^{\sigma} I_{(1)}^{\mu \lambda}\right)+\frac{1}{4} Q^{\mu} Q^{\sigma} I_{(0)}^{\lambda}\right)+(\rho \leftrightarrow \sigma) .
\end{aligned}
$$

Using the explicit results for the integrals in appendices B and C, and following the method outlined in the previous subsection, we find that the third order response function is still given by eq. (3.18), with the coefficients replaced by

$$
\begin{aligned}
& c_{\mathbb{V}}=\frac{\mu q^{2}}{192 \pi^{2}}\left[-\frac{2 Q^{2}}{q^{2}}+\frac{3 Q^{2}\left(q^{2}-2 Q^{2}\right)}{q^{4}} L\left(q^{0}, q\right)\right], \\
& c_{\mathbb{T}}=\frac{\mu q^{2}}{192 \pi^{2}}\left[\frac{Q^{4}}{2 q^{4}}+\frac{3 Q^{6}}{2 q^{6}} L\left(q^{0}, q\right)\right] .
\end{aligned}
$$

In the static limit they become

$$
\begin{aligned}
& c_{\mathbb{V}}(0, q)=\frac{\mu}{192 \pi^{2}} q^{2}, \\
& c_{\mathbb{T}}(0, q)=-\frac{\mu}{192 \pi^{2}} q^{2} .
\end{aligned}
$$

This completes the computation of the parity-odd response function to third order in $Q^{\nu}$.

\section{Energy-momentum tensor and metric perturbations}

In this section we will use the results obtained for the parity-odd response function to derive the general form of the parity violating part of the energy-momentum tensor. We will devote special attention to the static case, where our results can be compared with recent proposals in the literature. As shown above, the effects of metric perturbations on the energy-momentum tensor

$$
\delta\left\langle T^{\mu \nu}\right\rangle=-\frac{1}{2} \Pi^{\mu \nu \rho \sigma}\left(q^{0}, \boldsymbol{q}\right) h_{\rho \sigma},
$$

can be parametrized by the two independent functions $c_{\mathbb{V}}\left(q^{0}, q\right)$ and $c_{\mathbb{T}}\left(q^{0}, q\right)$

$$
\begin{aligned}
\Pi^{\mu \nu \rho \sigma}\left(q^{0}, \boldsymbol{q}\right)= & i c_{\mathbb{V}}\left(q^{0}, q\right) \frac{Q^{2}}{(u \cdot Q)^{2}+Q^{2}} u_{\alpha} Q_{\beta}\left[\epsilon^{\alpha \beta \mu \rho} P_{\mathbb{V}}^{\nu \sigma}+\epsilon^{\alpha \beta \nu \rho} P_{\mathbb{V}}^{\mu \sigma}+(\rho \leftrightarrow \sigma)\right] \\
& +i c_{\mathbb{T}}\left(q^{0}, q\right) u_{\alpha} Q_{\beta}\left[\epsilon^{\alpha \beta \mu \rho} P_{\mathbb{T}}^{\nu \sigma}+\epsilon^{\alpha \beta \nu \rho} P_{\mathbb{T}}^{\mu \sigma}+(\rho \leftrightarrow \sigma)\right] .
\end{aligned}
$$




\begin{tabular}{c||c|c|c} 
& Scalar & Vector & Tensor \\
\hline \hline$h_{00}$ & $-2 \sigma$ & - & - \\
$h_{0 i}$ & $-\partial_{i} b$ & $-a_{i}^{(S)}$ & - \\
$h_{i j}$ & $c \delta_{i j}+\partial_{i} \partial_{j} d$ & $\partial_{i} F_{j}+\partial_{j} F_{i}$ & $\tilde{h}_{i j}$ \\
\hline
\end{tabular}

Table 1. $S O(3)$ components of a general perturbation of the metric, where we have defined $a_{i}^{(S)}$ and $a_{i}^{(L)}=\partial_{i} b$ as the solenoidal and irrotational parts of $\delta g_{0 i}=-a_{i}(t, \boldsymbol{x})$.

It should be noted that, as a consequence of the form of $\Pi^{\mu \nu \rho \sigma}$, the response of the energy-momentum tensor depends only on gauge-invariant combinations of the metric disturbances. In order to simplify the analysis, a general metric perturbation has been decomposed into $S O(3)$ irreducible components in table 1 . Note that the vector fields $a_{i}^{(S)}$ and $F_{i}$ are solenoidal, while $\tilde{h}_{i k}$ is traceless and satisfies $\partial_{i} \tilde{h}_{i k}=0$. Direct substitution shows that scalar perturbations do not produce any parity-violating effect on $T^{\mu \nu}$. For vector perturbations, not necessarily time-independent, the change in the expectation value of $\left\langle T^{\mu \nu}\right\rangle$ depends only on the combination $a_{i}+\partial_{t} F_{i}$, which is gauge-invariant

$$
\begin{aligned}
\delta\left\langle T^{0 i}\right\rangle & =c_{\mathbb{V}}\left(q^{0}, q\right) i \epsilon^{i j k} q^{j}\left(-a_{k}+i q^{0} F_{k}\right), \\
\delta\left\langle T^{i j}\right\rangle & =c_{\mathbb{V}}\left(q^{0}, q\right) i q^{0}\left(\epsilon^{i m n} \hat{q}^{m} \hat{q}^{j}+\epsilon^{j m n} \hat{q}^{m} \hat{q}^{i}\right)\left(-a_{n}+i q^{0} F_{n}\right),
\end{aligned}
$$

where $\hat{q}^{j}=q^{j} / q$. Thus, $c \mathbb{V}\left(q^{0}, q\right)$ parametrizes the response to vector perturbations of the metric. Similarly, $c_{\mathbb{T}}\left(q^{0}, q\right)$ parametrizes the response to tensor perturbations $\tilde{h}_{i j}$, which are gauge-invariant by construction

$$
\delta\left\langle T^{i j}\right\rangle=-c_{\mathbb{T}}\left(q^{0}, q\right) \epsilon^{i l m} \delta^{j n} i q^{l} \tilde{h}_{m n}+(i \leftrightarrow j) .
$$

These are the main results in this paper. In what follows, we will compare them with other results in the literature.

\subsection{Static limit of response functions and anomalous constitutive relations}

Arguments based on linearized hydrodynamics [16] show that the small velocity field of the fluid $v^{j}(t, \boldsymbol{x})$ is the quantity that plays the role of external force coupled to the momentum density $T^{0 i}$ in the perturbing Hamiltonian

$$
H^{\mathrm{ext}}=-\int d^{3} \boldsymbol{x} T^{0 i}(t, \boldsymbol{x}) v^{i}(t, \boldsymbol{x}) .
$$

Hence a comparison with

$$
T^{\mu \nu}=\frac{2}{\sqrt{-g}} \frac{\delta S}{\delta g_{\mu \nu}},
$$

enables us to identify $h_{0 i}=-a_{i}$ with the fluid velocity $v^{i}$ in the static limit $q^{0}=0$. Thus, using (3.20) we find the following constitutive relations in the static limit at leading order in the momenta

$$
\begin{aligned}
& \delta\left\langle T^{0 i}\right\rangle=c_{\mathbb{V}}(0, q) \epsilon^{i j k} i q^{j} v^{k}, \\
& \delta\left\langle T^{i j}\right\rangle=0 .
\end{aligned}
$$


We see that $c_{\mathbb{V}}(0, q)$ may be interpreted as a parity-violating susceptibility connecting the vorticity with the momentum density. In fact, in hydrodynamics with quantum anomalies, the momentum density to linear order in $\boldsymbol{v}[14]$ is precisely given by

$$
\boldsymbol{g}=(\varepsilon+\mathcal{P}) \boldsymbol{v}+\chi_{\mathbb{V}} \boldsymbol{\nabla} \times \boldsymbol{v},
$$

when one uses a frame where the entropy current does not have anomalous part i.e., where $J_{S}^{\mu}=s u^{\mu}$. This agrees with our result (4.7) which, by (3.20), implies the following value for the anomalous susceptibility

$$
\chi_{\mathbb{V}}=c_{\mathbb{V}}(0, q)=-\frac{1}{12 \pi^{2}}\left(\mu^{3}+\pi^{2} \mu T^{2}\right) .
$$

Actually, the value of the parity violating susceptibility has been related to the gauge and mixed anomaly coefficients in the anomalous conservation equations

$$
\begin{aligned}
& \nabla_{\mu} J_{\mathrm{cov}}^{\mu}=\frac{1}{4} \epsilon^{\mu \nu \rho \sigma}\left(3 c_{A} F_{\mu \nu} F_{\rho \sigma}+c_{m} R_{\beta \mu \nu}^{\alpha} R_{\alpha \rho \sigma}^{\beta}\right), \\
& \nabla_{\nu} T_{\mathrm{cov}}^{\mu \nu}=F_{\nu}^{\mu} J_{\mathrm{Cov}}^{\nu}+\frac{1}{2} c_{m} \nabla_{\nu}\left(\epsilon^{\rho \sigma \alpha \beta} F_{\rho \sigma} R^{\mu \nu}{ }_{\alpha \beta}\right),
\end{aligned}
$$

through the relations [11]

$$
\chi_{\mathbb{V}}=2\left(\tilde{c}_{4 d} \mu T^{2}-c_{A} \mu^{3}\right), \quad \tilde{c}_{4 d}=-8 \pi^{2} c_{m} .
$$

The values of the anomaly coefficients for a left-handed spinor in $(3+1)$ dimensions,

$$
c_{A}=8 c_{m}=\frac{1}{24 \pi^{2}},
$$

then imply our value (4.9) for the anomalous susceptibility.

Similarly, we may use (3.26) to find the following $\mathcal{O}\left(Q^{3}\right)$ corrections to the constitutive relations in the static limit

$$
\begin{aligned}
\delta\left\langle T^{0 i}\right\rangle & =\frac{\mu}{192 \pi^{2}} \epsilon^{i j k} \nabla^{2} \partial_{j} a_{k}, \\
\delta\left\langle T^{i j}\right\rangle & =-\frac{\mu}{192 \pi^{2}} \epsilon^{i l m} \delta^{j k} \nabla^{2} \partial_{l} \tilde{h}_{k m}+(i \leftrightarrow j) .
\end{aligned}
$$

Note that (4.14) simply gives a correction to the momentum density (4.8)

$$
\delta \boldsymbol{g}=-\frac{\mu}{192 \pi^{2}} \nabla^{2}(\boldsymbol{\nabla} \times \boldsymbol{v})=-c_{m} \mu \nabla^{2}(\boldsymbol{\nabla} \times \boldsymbol{v}) .
$$

On the other hand, (4.15) describes a qualitatively new effect. For a tensorial perturbation depending only on $z$

$$
\tilde{h}_{i j}=\left(\begin{array}{ccc}
h_{+}(z) & h_{\times}(z) & 0 \\
h_{\times}(z) & -h_{+}(z) & 0 \\
0 & 0 & 0
\end{array}\right),
$$

eq. (4.15) gives

$$
\begin{aligned}
& T^{11}=-T^{22}=2 c_{m} \mu h_{\times}^{\prime \prime \prime}(z), \\
& T^{12}=-2 c_{m} \mu h_{+}^{\prime \prime \prime}(z) .
\end{aligned}
$$


Predictions for the effects of gauge and mixed anomalies on the constitutive relations at higher orders order in the momenta have only recently been given in $(1+1)$ [10] and $(3+1)$ dimensions [11]. In order to connect our results with those in [11] we collect some of their formulae. Using a frame where the energy-momentum tensor takes the form

$$
\left\langle T^{\mu \nu}\right\rangle=(\varepsilon+P) u^{\mu} u^{\nu}+P g^{\mu \nu}+u^{\mu} q_{A}^{\nu}+u^{\nu} q_{A}^{\mu}+\tau_{A}^{\mu \nu},
$$

with $u_{\mu} q_{A}^{\mu}=u_{\mu} \tau_{A}^{\mu \nu}=\tau_{A \mu}^{\mu}=0$, the following results were obtained [11] at $\mathcal{O}\left(Q^{3}\right)$ in $(3+1)$ dimensions

$$
\begin{aligned}
q_{A}^{\mu} & =-2 \mu c_{m} \tilde{v}_{3}^{\mu}=-2 \mu c_{m} \epsilon^{\mu \nu \rho \sigma} u_{\nu} \nabla_{\rho} R_{\sigma \alpha} u^{\alpha}+\ldots \\
\tau_{A}^{\mu \nu} & =4 \mu c_{m} \tilde{t}_{3}^{\mu \nu}=4 \mu c_{m} \Delta^{\alpha<\mu} \epsilon^{\nu>\rho \sigma \beta} u_{\rho} \nabla_{\sigma} R_{\alpha \beta}+\ldots
\end{aligned}
$$

where the dots stand for contributions that vanish at linear order in the metric perturbations and are thus invisible in our computation. In this formula

$$
V^{<\mu \nu>} \equiv \Delta^{\mu \rho} \Delta^{\nu \sigma} V_{(\mu \nu)}-\frac{1}{3} \Delta^{\mu \nu} \Delta_{\rho \sigma} V^{\rho \sigma}
$$

and $\Delta^{\mu \nu}=g^{\mu \nu}+u^{\mu} u^{\nu}$. In order to compare with (4.14), we evaluate the pseudovector $\tilde{v}_{3}^{\mu}$ at linear order in the metric perturbation. This yields $\tilde{v}_{3}^{0}=0$ and

$$
\tilde{v}_{3}^{i}=-\frac{1}{2} \epsilon^{i j k}\left(\partial_{j} \nabla^{2} h_{k 0}-\partial_{0} \partial_{j} \partial_{l} h_{l k}\right)+\mathcal{O}\left(h^{2}\right),
$$

where we have taken $u^{\mu}=\delta_{0}^{\mu}+\mathcal{O}(h)$. Upon substitution in this expression of the metric components in table 1 we obtain

$$
q_{A}^{i}=-2 \mu c_{m} \tilde{v}_{3}^{i}=-\frac{\mu}{192 \pi^{2}} \epsilon^{i j k} \nabla^{2} \partial_{j}\left(a_{k}+\partial_{t} F_{k}\right)+\mathcal{O}\left(h^{2}\right),
$$

which differs in sign from our contribution (4.14) to $\delta\left\langle T^{0 i}\right\rangle$ in the static limit. On the other hand, note that precisely the combination $a_{k}+\partial_{t} F_{k}$, which we have argued should be identified with the fluid velocity, appears in this expression. Proceeding similarly with the pseudotensor gives the non-vanishing components

$$
\tilde{t}_{3}^{i j}=-\frac{1}{4} \epsilon^{i l m}\left(\partial_{l} \partial^{\alpha} \partial_{\alpha} h_{m}{ }^{j}-\partial_{l} \partial^{j} \partial_{\alpha} h_{m}{ }^{\alpha}\right)+(i \leftrightarrow j)+\mathcal{O}\left(h^{2}\right)
$$

Taking the static limit and substituting the metric components in table 1 gives

$$
\tau_{A}^{i j}=4 \mu c_{m} \tilde{t}_{3}^{i j}=-\frac{\mu}{192 \pi^{2}} \epsilon^{i l m} \nabla^{2} \partial_{l} \tilde{h}_{m}^{j}+(i \leftrightarrow j),
$$

which exactly agrees with (4.15). In the next section we will explore the relations of our linearized results with the full anomalous partition function given in [11] and will be able to explain the sign discrepancy noted above. 


\section{The anomalous partition function and the static response function}

In this section we consider in more detail how our previous results for the static response functions fit with known facts about gauge and gravitational anomalies of the underlying theory, particularly with the form of the full anomalous partition function. As we will see, an important feature of the third order response (3.26) is that it reveals the existence of an invariant contribution to the partition function which, in principle, does not seem to arise from general arguments about anomalies.

We will assume that an external time-independent gauge field $\mathcal{A}=\mathcal{A}_{0}(\boldsymbol{x}) d t+\mathcal{A}_{i}(\boldsymbol{x}) d x^{i}$ is present besides the static gravitational field given in (2.9). In this background with Killing vector $V^{\mu}=(1, \mathbf{0})$, the equilibrium temperature and chemical potential become position-dependent. They are defined in terms of the invariant length of the time circle and the Polyakov loop $P_{A}$ as [7]

$$
\begin{aligned}
T(\boldsymbol{x})^{-1} & =\int_{0}^{1 / T_{0}} d \tau \sqrt{-V^{\mu} V_{\mu}}=\frac{e^{\sigma}}{T_{0}}, \\
\frac{\mu(\boldsymbol{x})}{T(\boldsymbol{x})} & =\ln P_{A}=\int_{0}^{1 / T_{0}} d \tau \mathcal{A}_{\mu}(\boldsymbol{x}) V^{\mu}=\frac{\mathcal{A}_{0}(\boldsymbol{x})}{T_{0}},
\end{aligned}
$$

where $T_{0}^{-1}$ is the length of the compactified imaginary time.

\subsection{The first order generating functional}

Let us start with the first order in the derivative expansion. Using the definition of the energy-momentum tensor in terms of the partition function ${ }^{3}$

$$
T^{\mu \nu}=\frac{2 T_{0}}{\sqrt{-g}} \frac{\delta W}{\delta g_{\mu \nu}(\boldsymbol{x})},
$$

the linearized constitutive relation (4.7) may be rewritten in terms of $\mathcal{A}_{0}$ and $T_{0}$ (or equivalently $\mu$ and $T$ )

$$
\delta\left\langle T^{0 i}\right\rangle=-T_{0} \frac{\delta W}{\delta a_{i}}=\left(2 c_{A} \mathcal{A}_{0}^{3}-2 \tilde{c}_{4 d} T_{0}^{2} \mathcal{A}_{0}\right) \tilde{\epsilon}^{i j k} \partial_{j} a_{k}+\ldots,
$$

where $\tilde{\epsilon}^{123}=1$, and $\epsilon^{i j k}=\tilde{\epsilon}^{i j k} / \sqrt{g_{3}}$ will denote the corresponding tensor. Since the quantities $a_{j}$ and $\mathcal{A}_{i}$ transform like the components of a covariant vector under spatial diffeomorphisms and $\mathcal{A}_{0}$ behaves like a scalar, it is clear that $W$ must include the terms

$$
-\frac{c_{A}}{T_{0}} \int d^{3} x \sqrt{g_{3}} \epsilon^{i j k} \mathcal{A}_{0}^{3} a_{i} \partial_{j} a_{k}+\tilde{c}_{4 d} T_{0} \int d^{3} x \sqrt{g_{3}} \epsilon^{i j k} \mathcal{A}_{0} a_{i} \partial_{j} a_{k} .
$$

To reconstruct the full dependence of $W$ on the gauge field, we complete the action with the requirements of gauge invariance up to a $U(1)$ anomaly, and invariance under Kaluza-Klein transformations [9]. These correspond to redefinitions of time, $t \rightarrow t^{\prime}=t+\phi(\boldsymbol{x})$, without change in the spatial coordinate, and preserve the form of the metric if $a_{i}$ transforms

\footnotetext{
${ }^{3}$ Here $W=\ln Z=-\Omega / T_{0}$, where $Z$ is the grand partition function and $\Omega$ refers to the thermodynamic potential, a functional of the background quantities depending solely on $\boldsymbol{x}$.
} 
as $\delta a_{i}=-\partial_{i} \phi$. Under such a transformation the gauge field changes as $\delta \mathcal{A}_{0}=0, \delta \mathcal{A}_{i}=$ $-\mathcal{L}_{\phi} \mathcal{A}_{i}=-\mathcal{A}_{0} \partial_{i} \phi$, and the combination $\mathcal{A}_{i}-a_{i} \mathcal{A}_{0}$ remains invariant. As this kind of gauge invariance may be viewed as a manifestation of the underlying diffeomorphism invariance, which is not anomalous at the first derivative order, it is natural to impose this requirement on the partition function. A short computation shows that, up to a total derivative, the resulting Kaluza-Klein invariant action is given by

$$
\begin{aligned}
W= & -\frac{c_{A}}{T_{0}} \int d^{3} x \sqrt{g_{3}} \epsilon^{i j k} \mathcal{A}_{0}^{3} a_{i} \partial_{j} a_{k}+\frac{3 c_{A}}{T_{0}} \int d^{3} \sqrt{g_{3}} x \epsilon^{i j k} \mathcal{A}_{0}^{2} \mathcal{A}_{i} \partial_{j} a_{k} \\
& -\frac{2 c_{A}}{T_{0}} \int d^{3} x \sqrt{g_{3}} \epsilon^{i j k} \mathcal{A}_{0} \mathcal{A}_{i} \partial_{j} \mathcal{A}_{k}-\tilde{c}_{4 d} T_{0} \int d^{3} x \sqrt{g_{3}} \epsilon^{i j k}\left(\mathcal{A}_{i}-\mathcal{A}_{0} a_{i}\right) \partial_{j} a_{k} .
\end{aligned}
$$

Thus the static gravitational response, together with the requirements of invariance under Kaluza-Klein transformations and gauge invariance up to a $U(1)$ anomaly, determine the partition function at first order in the derivative expansion. The first three terms in (5.5) constitute the anomalous part of the consistent partition function, while the last one is gauge invariant. Our result (5.5) agrees with the form of the parity-odd partition function proposed in [9] for a CPT invariant theory. The consistent gauge anomaly follows from the variation of this action under a gauge transformation $\delta \mathcal{A}_{i}=\partial_{i} \Lambda, \delta \mathcal{A}_{0}=0$. This induces a change

$$
\delta W=\frac{2 c_{A}}{T_{0}} \int d^{3} x \sqrt{g_{3}} \Lambda \epsilon^{i j k} \partial_{i} \mathcal{A}_{0} \partial_{j} \mathcal{A}_{k}
$$

which shows that the consistent anomaly is precisely determined by the cubic term in the static momentum density correlator. The variation of the consistent current under such a gauge transformation is given by

$$
\begin{aligned}
\delta J^{0} & =-2 c_{A} \frac{\tilde{\epsilon}^{i j k}}{\sqrt{-g}} \partial_{j} \mathcal{A}_{k} \partial_{i} \Lambda, \\
\delta J^{i} & =-2 c_{A} \frac{\tilde{\epsilon}^{i j k}}{\sqrt{-g}} \partial_{j} \mathcal{A}_{0} \partial_{k} \Lambda .
\end{aligned}
$$

Therefore the anomalous gauge invariant current is obtained by the compensating shift

$$
J_{\text {cov }}^{\mu}=\frac{T_{0}}{\sqrt{-g}} \frac{\delta W}{\delta \mathcal{A}_{\mu}}+2 c_{A} \epsilon^{\mu \nu \rho \sigma} \mathcal{A}_{\nu} \partial_{\rho} \mathcal{A}_{\sigma}
$$

Note that the Bardeen-Zumino term in this equation contributes to the current at first order in the derivative expansion.

\subsection{The third order generating functional}

Now we turn our attention to the connection between the static gravitational response at third order in the derivative expansion and the mixed anomaly. First, we introduce the Pontryagin density defined by

$$
\mathcal{P}=\frac{1}{2}{ }^{*} R_{\nu}^{\mu}{ }^{\alpha \beta} R_{\mu \alpha \beta}^{\nu},
$$

where the dual Riemann tensor is given by

$$
{ }^{*} R_{\nu}^{\mu}{ }_{\nu}^{\alpha \beta}=\frac{1}{2} \epsilon^{\alpha \beta \rho \tau} R_{\nu \rho \tau}^{\mu} .
$$


Locally, the Pontryagin density can be written as a total divergence

$$
\nabla_{\mu} K^{\mu}=\mathcal{P}
$$

where $K^{\mu}$ is the Chern-Simons topological current

$$
K^{\alpha}=\epsilon^{\alpha \beta \rho \tau} \Gamma_{\beta \eta}^{\xi}\left(\partial_{\rho} \Gamma_{\tau \xi}^{\eta}+\frac{2}{3} \Gamma_{\rho \delta}^{\eta} \Gamma_{\tau \xi}^{\delta}\right) .
$$

We have seen from (4.15) that the response to a tensor perturbation of the form (4.17) is given by

$$
\begin{aligned}
& T^{11}=-T^{22}=2 c_{m} \mu h_{\times}^{\prime \prime \prime}(z), \\
& T^{12}=-2 c_{m} \mu h_{+}^{\prime \prime \prime}(z) .
\end{aligned}
$$

This response involving only spatial indices may actually be derived from the quadratic portion of the functional ${ }^{4}$

$$
W_{K}=-\frac{c_{m}}{T_{0}} \int d^{3} x \sqrt{-g} \mathcal{A}_{\mu} K^{\mu}
$$

which, for $\mathcal{A}_{0}=\mu, \mathcal{A}_{i}=0$, becomes

$$
W_{K}^{\text {quad }}=-\frac{c_{m}}{T_{0}} \int d^{3} x \mu\left(h_{+}^{\prime}(z) h_{\times}^{\prime \prime}(z)-h_{+}^{\prime \prime}(z) h_{\times}^{\prime}(z)\right) .
$$

But $W_{K}^{\text {quad }}$, when evaluated for the vector perturbation $h_{01}=-a_{1}(z), h_{02}=-a_{2}(z)$, $h_{03}=0$,

$$
W_{K}^{\text {quad }}=-\frac{c_{m}}{T_{0}} \int d^{3} x \mu K_{\text {quad }}^{0}=-\frac{c_{m}}{2 T_{0}} \int d^{3} x \mu\left(a_{2}^{\prime}(z) a_{1}^{\prime \prime}(z)-a_{1}^{\prime}(z) a_{2}^{\prime \prime}(z)\right),
$$

does not produce the required response. Indeed its variation yields

$$
\begin{aligned}
& T^{01}=-T_{0} \frac{\delta W_{K}^{\text {quad }}}{\delta a_{1}}=c_{m} \mu a_{2}^{\prime \prime \prime}(z), \\
& T^{02}=-T_{0} \frac{\delta W_{K}^{\text {quad }}}{\delta a_{2}}=-c_{m} \mu a_{1}^{\prime \prime \prime}(z),
\end{aligned}
$$

which is minus our result (4.14). This is consistent with our observations at the end of last section. Therefore, an additional contribution to the action will be needed in order to properly account for the vector response. The following is a natural, possibly unique choice that preserves $U(1)$ gauge invariance, three-dimensional diffeomorphism invariance and Kaluza-Klein gauge invariance

$$
W_{\mathrm{inv}}=\frac{c_{1}}{T_{0}} \int d^{3} x \sqrt{g_{3}} \epsilon^{i j k} g_{i n} \frac{1}{\sqrt{g_{3}}} \partial_{m}\left(\sqrt{g_{3}} f^{m n}\right) \partial_{j}\left(\mathcal{A}_{k}-a_{k} \mathcal{A}_{0}\right)
$$

\footnotetext{
${ }^{4}$ In the linear approximation the required components read $T^{11}=T_{0} \delta W_{K}^{\text {quad }} / \delta h_{+}=-T^{22}$ and $T^{12}=$ $T_{0} \delta W_{K}^{\text {quad }} / \delta h_{\times}$.
} 
where the inverse metric $g^{k l}$ is used to raise the lower indices of $f_{i j} \equiv \partial_{i} a_{j}-\partial_{j} a_{i}$, and $c_{1}$ is a constant to be determined shortly. In terms of differential forms the integrand is proportional to $d\left(\mathcal{A}-\mathcal{A}_{0} a\right) \wedge \delta d a$, where $\delta$ denotes the codifferential. The virtue of $W_{\text {inv }}$ is that the linearized tensor response remains unaffected, while its contribution the vector response reads

$$
\begin{aligned}
& -T_{0} \frac{\delta W_{\mathrm{inv}}^{(2)}}{\delta a_{1}}=-2 c_{1} \mu a_{2}^{\prime \prime \prime}(z), \\
& -T_{0} \frac{\delta W_{\text {inv }}^{(2)}}{\delta a_{2}}=2 c_{1} \mu a_{1}^{\prime \prime \prime}(z) .
\end{aligned}
$$

If we could identify $W_{K}$ with the anomalous partition function, then $c_{1}=c_{m}$ would be the appropriate choice in order to reproduce our linearized results (4.14). However, $W_{K}$ can not be identified with the partition function. Instead, the non-invariant functional $W_{K}$ plays the role of a local counterterm that interpolates between two alternative definitions of the consistent effective action. These two choices preserve either diffeomorphism or gauge invariance. Following Bilal's notation [13], if we denote by $\Gamma^{(1)}$ the diffeormorphism invariant effective action for the mixed gauge-gravitational anomaly, and by $\Gamma^{(2)}$ the gauge invariant one, the interpolation is given by

$$
W_{K}+\Gamma^{(1)}=\Gamma^{(2)} .
$$

We must then consider the contributions to the energy-momentum tensor from $\Gamma^{(1,2)}$ and the relation with our results for the static response.

The complete anomalous contribution to the partition function at third derivative order, together with its implications on the constitutive relations, have been recently stablished in [11]. With the vorticity and acceleration of the fluid given by

$$
\begin{aligned}
\omega^{\mu} & =\epsilon^{\mu \nu \rho \sigma} u_{\nu} \nabla_{\rho} u_{\sigma}, \\
a^{\mu} & =u^{\lambda} \nabla_{\lambda} u^{\mu},
\end{aligned}
$$

the authors of [11] construct the well-behaved covariant current

$$
j_{m}^{\mu}=-4 W_{\nu \rho \sigma}^{\mu} u^{\nu} u^{\rho} \omega^{\sigma}-\left(\frac{1}{3} R+2 R_{\rho \sigma} u^{\rho} u^{\sigma}-2 a^{\lambda} a_{\lambda}-\frac{3}{2} \omega^{\lambda} \omega_{\lambda}\right) \omega^{\mu},
$$

where $W_{\nu \rho \sigma}^{\mu}$ is the Weyl tensor and $R_{\rho \sigma}$ is the Ricci tensor. This current, evaluated for the equilibrium fluid velocity $u^{\mu}=e^{-\sigma} V^{\mu}$ in the background (2.9), has the same divergence as the topological current

$$
\nabla_{\mu} j_{m}^{\mu}=\mathcal{P} .
$$

This implies that the local functionals

$$
\begin{aligned}
\Gamma^{(1)} & =\frac{c_{m}}{T_{0}} \int d^{3} x \sqrt{-g} \mathcal{A}_{\mu} j_{m}^{\mu}, \\
\Gamma^{(2)} & =\frac{c_{m}}{T_{0}} \int d^{3} x \sqrt{-g} \mathcal{A}_{\mu}\left(j_{m}^{\mu}-K^{\mu}\right),
\end{aligned}
$$


satisfy (5.20) and have the required properties: $\Gamma^{(1)}$ is obviously invariant under threedimensional diffeomorphisms, but not gauge invariant, whereas, thanks to $(5.23), \Gamma^{(2)}$ is gauge invariant under $\delta \mathcal{A}_{i}=\partial_{i} \Lambda, \delta \mathcal{A}_{0}=0$, but not diffeomorphism invariant. As a consequence, these functionals give rise to two different types of consistent observables.

Let's first consider the combination $\Gamma^{(2)}+W_{\text {inv }}$. It is easy to see that the component $j_{m}^{0}$ is cubic in the metric perturbation and does not contribute to the linearized $T^{\mu \nu}$. Thus, for $\mathcal{A}_{0}$ constant and $\mathcal{A}_{i}=0$, the quadratic part of $\Gamma^{(2)}$ matches that of $W_{K}$, and has identical contributions to the linearized $T^{\mu \nu}$. On the other hand, the Bardeen-Zumino term required to compensate for the lack of invariance under diffeomorphisms vanishes if the gauge field strength $\mathcal{F}_{\alpha \beta}$ does [11], and the linear response results will agree with the variation of the quadratic part of $\Gamma^{(2)}+W_{\mathrm{inv}}$, which coincides with that of $W_{K}+W_{\mathrm{inv}}$, for the choice $c_{1}=c_{m}$.

For a different choice of the generating functional, namely for $\Gamma^{(1)}+W_{\text {inv }}$, the conclusions are identical, although they require a little more work. This functional may be viewed as the third derivative counterpart of the first derivative action (5.5), since both of them are diffeomorhism invariant. Differentiation with respect to $\mathcal{A}_{\mu}$ yields a gauge and diffeomorfism covariant anomalous current with no need for a compensating term. But, while the first derivative term $\delta W / \delta g_{\mu \nu}$ from (5.5) was gauge invariant, the consistent contribution $\delta\left(\Gamma^{(1)}+W_{\text {inv }}\right) / \delta g_{\mu \nu}$ is not, and a Bardeen-Zumino tensor $T_{B Z}^{\mu \nu}$ has to be added in order to produce a gauge invariant and generally covariant energy-momentum tensor

$$
T^{\mu \nu}=\frac{2 T_{0}}{\sqrt{-g}} \frac{\delta\left(\Gamma^{(1)}+W_{\mathrm{inv}}\right)}{\delta g_{\mu \nu}}+T_{B Z}^{\mu \nu} .
$$

As $j_{m}^{0}$ is cubic in the metric perturbation, $\Gamma^{(1)}$ does not contribute to the linearized $T^{\mu \nu}$. Thus the third-order derivative response, which obeys the Ward identity of general covariance, must precisely match the linear portion of the Bardeen-Zumino tensor, together with the contribution from $W_{\text {inv }}$. This turns out to be the case if $c_{1}$ takes the value $c_{1}=c_{m}$, which is determined from (5.19). Indeed, an explicit computation shows that the results in (4.14) and (4.15) may be rewritten as

$$
\delta\left\langle T^{\mu \nu}\right\rangle=2 T_{0} \frac{\delta W_{\text {inv }}^{(2)}}{\delta h_{\mu \nu}}+2 c_{m} \partial_{j}\left({ }^{*} R_{\text {lin }}^{j \mu \nu 0} \mu_{0}+{ }^{*} R_{\text {lin }}^{j \nu \mu 0} \mu_{0}\right) .
$$

The non-linear generalization of the second term,

$$
T_{B Z}^{\mu \nu}=2 c_{m} \nabla_{\lambda}\left({ }^{*} R^{\lambda \mu \nu \rho} \mathcal{A}_{\rho}+{ }^{*} R^{\lambda \nu \mu \rho} \mathcal{A}_{\rho}\right)
$$

exactly matches the form of the Bardeen-Zumino energy tensor given in [11] when the anomalous piece of the generating functional is precisely $\Gamma^{(1)}$.

Thus the results at the third derivative order obtained in this paper show that a consistent generating functional describing the effects of the mixed anomaly must include an additional invariant piece $W_{\text {inv }}$ given by (5.18) with $c_{1}=c_{m}$, a feature that ultimately can be traced to the opposite signs for $c_{\mathbb{V}}$ and $c_{\mathbb{T}}$ in (3.26). 


\section{Discussion and outlook}

In this paper we have computed the subleading corrections to the time-dependent parity violating graviton response function up to third order in the derivative expansion. In particular, we have obtained the complete invariant decomposition of the response function on the appropriate tensor basis (3.14) and (C.1). We have then exploited the transversality of the response function to rewrite our results in terms of the two invariant functions $c_{\mathbb{V}, \mathbb{T}}$ which condense all the relevant information.

From the static limit of the response function we have extracted the anomalous constitutive relations at first (4.7), and third order (4.14), (4.15) in the derivative expansion, which we have compared with recent results in the literature. We have also shown that one can reconstruct the complete parity violating partition function at first order in the derivative expansion (5.5) from the linearized corrections to the energy-momentum tensor (4.7). The situation at third order in the derivative expansion is more involved, but we have shown that our expressions for the constitutive relations are fully compatible with very recent results on the form of the anomalous partition function at that order if one includes the previously unknown parity-odd invariant contribution given by (5.18).

We have also obtained the complete dependence of the quantities $c_{\mathbb{V}, \mathbb{T}}$ on $q^{0} / q$, from which one can compute chiral effects on the time-dependent departures from equilibrium $\delta\left\langle T^{\mu \nu}(t, \boldsymbol{q})\right\rangle$. For the parity-even part of the time-dependent response to metric perturbations, an analysis including a comparison with the results from the Boltzmann equation has been given in [20]. Now, having at our disposal a set of results for the parity-odd part of the response, we can pose the question about the relationship between the field theory approach presented in this paper and a possible kinetic description involving the background metric. In this regard, the modification of the non-equilibrium kinetic equation that take into account chiral magnetic and anomalous Hall effects has been recently obtained in $[21,22]$ in the presence of an external electromagnetic field. In addition to this, it would be interesting to take advantage of the results at the linear level in this paper to obtain a Vlasov-type equation from which one could derive non-equilibrium chiral effects caused by metric perturbations or weak curvature backgrounds. We leave the consideration of this issues for future work ${ }^{5}$.

\section{Acknowledgments}

This work is supported in part by the Spanish Ministry of Science and Technology under Grant FPA2009-10612 and the Spanish Consolider-Ingenio 2010 Programme CPAN (CSD2007-00042), and by the Basque Government under Grant IT559-10.

\section{A Expansion of the action and seagull terms}

In this appendix we obtain eq. (3.5) for the seagull contribution to the parity-odd part of the response function. The action for a fermion in a curved background is given by

\footnotetext{
${ }^{5}$ After this work was completed, we became aware of [23], which deals with issues similar to the ones mentioned in this paragraph.
} 
$S=\int d^{4} x \sqrt{-g} \mathcal{L}$ where

$$
\mathcal{L}=\frac{i}{2}\left[\bar{\psi} \gamma^{\mu} \nabla_{\mu} \psi-\left(\nabla_{\mu} \bar{\psi}\right) \gamma^{\mu} \psi\right]
$$

with $\nabla_{\mu} \psi=\partial_{\mu} \psi-\Gamma_{\mu} \psi$. The spin connection is related to the vierbein $e_{a}^{\nu}$ by

$$
\Gamma_{\mu}=\frac{1}{8}\left[\gamma^{a}, \gamma^{b}\right] e_{a}^{\nu} e_{b \nu ; \mu}=\frac{1}{8}\left[\gamma^{a}, \gamma^{b}\right] e_{a}^{\nu}\left(\partial_{\mu} e_{b \nu}-\Gamma_{\mu \nu}^{\alpha}\right) e_{\beta \alpha},
$$

where greek and latin letters are used for curved and Minkowski indices respectively, with $\left\{\gamma^{\mu}, \gamma^{\nu}\right\}=-2 g^{\mu \nu}$ and $\left\{\gamma^{a}, \gamma^{b}\right\}=-2 \eta^{a b}$. Expanding the action $\mathcal{S}=\int d^{4} x \mathcal{L}$ in powers of $h_{\mu \nu}=g_{\mu \nu}-\eta_{\mu \nu}$ gives $\mathcal{S}=\mathcal{S}_{0}+\mathcal{S}_{1}+\mathcal{S}_{2}+\ldots$ where $\mathcal{S}_{0}$ is the action in flat space-time, $\mathcal{S}_{1}=\frac{1}{2} \int d^{4} x T^{\mu \nu} h_{\mu \nu}$ with

$$
T_{\mu \nu}=\frac{i}{4}\left[\bar{\psi} \gamma_{\mu} \overleftrightarrow{\partial}_{\nu} \psi+\bar{\psi} \gamma_{\nu} \overleftrightarrow{\partial}_{\mu} \psi\right]-\frac{i}{2} \eta_{\mu \nu} \bar{\psi} \gamma^{\alpha} \overleftrightarrow{\partial}_{\alpha} \psi
$$

and

$$
\begin{aligned}
\mathcal{S}_{2}= & \frac{i}{16} \int d^{4} x \bar{\psi}\left\{\sigma^{\mu \nu}, \gamma^{\rho}\right\} \psi \eta^{\alpha \beta} h_{\alpha \mu} \partial_{\rho} h_{\beta \nu} \\
& -\frac{1}{8} \int d^{4} x\left(3 \eta^{\alpha \beta} T^{\mu \nu}-2 \eta^{\alpha \mu} T^{\beta \nu}\right) h_{\alpha \mu} h_{\beta \nu} \\
& +\frac{1}{8} \int d^{4} x\left(\eta^{\alpha \beta} \eta^{\mu \nu}-\eta^{\alpha \mu} \eta^{\beta \nu}\right) \mathcal{L} h_{\alpha \mu} h_{\beta \nu},
\end{aligned}
$$

with $\sigma^{\nu \sigma} \equiv \frac{1}{4}\left[\gamma^{\nu}, \gamma^{\sigma}\right]$. The three-vertex fermion-fermion-graviton in eq. (3.4) then follows from the Fourier transform of eq. (A.3). Similarly, the seagull contribution in eq. (3.5) follows from eq. (A.4) through

$$
\begin{aligned}
& \Pi_{2}^{\mu \nu \rho \sigma}(Q)=-4\left\langle\frac{\delta^{2} \mathcal{S}_{2}}{\delta h_{\mu \nu} \delta h_{\rho \sigma}}\right\rangle=\frac{1}{8} \eta^{\mu \rho} \frac{1}{\beta} \sum_{\omega_{n}} \int \frac{d^{3} k}{(2 \pi)^{3}} \operatorname{tr}\left[\left\{\sigma^{\nu \sigma}, \not \ell\right\} \mathcal{P}_{-} \not K\right] \frac{1}{K^{2}} \\
& +\frac{1}{8} \eta^{\nu \rho} \frac{1}{\beta} \sum_{\omega_{n}} \int \frac{d^{3} k}{(2 \pi)^{3}} \operatorname{tr}\left[\left\{\sigma^{\mu \sigma}, \not \subset\right\} \mathcal{P}_{-} \mathbb{K}_{K}\right] \frac{1}{K^{2}} \\
& +\frac{3}{4}\left(\eta^{\mu \rho}\left\langle T^{\sigma \nu}\right\rangle+\eta^{\nu \rho}\left\langle T^{\sigma \mu}\right\rangle\right)+(\rho \leftrightarrow \sigma) \\
& -\left(\eta^{\mu \nu}\left\langle T^{\rho \sigma}\right\rangle+\eta^{\rho \sigma}\left\langle T^{\mu \nu}\right\rangle\right),
\end{aligned}
$$

where we have used the fact that the the equations of motion imply $\langle\mathcal{L}\rangle=0$. As $\left\langle T^{\mu \nu}\right\rangle$ in flat space-time takes the form (2.8), which obviously preserves parity, this establishes the validity of eq. (3.5).

\section{B The integrals $I^{\mu \nu \rho}$}

Table 2 gives the contractions of $I^{\mu \nu \rho}$ with the basis tensors in eq. (3.14). The constants $I_{(n)}$ are related to the Fermi distribution as follows

$$
\begin{aligned}
& I_{(0)}=\int_{0}^{\infty} \frac{d k}{4 \pi^{2}} k^{2}\left(n_{F}(k-\mu)-n_{F}(k+\mu)\right)=\frac{1}{12 \pi^{2}}\left(\mu^{3}+\pi^{2} \mu T^{2}\right), \\
& I_{(1)}=\frac{1}{4} \int_{0}^{\infty} \frac{d k}{4 \pi^{2}} k\left(n_{F}(k-\mu)+n_{F}(k+\mu)\right)=\frac{1}{96 \pi^{2}}\left(3 \mu^{2}+\pi^{2} T^{2}\right), \\
& I_{(2)}=\frac{1}{48} \int_{0}^{\infty} \frac{d k}{4 \pi^{2}}\left(n_{F}(k-\mu)-n_{F}(k+\mu)\right)=\frac{\mu}{192 \pi^{2}},
\end{aligned}
$$




\begin{tabular}{c||c|c|c} 
& $n=0$ & $n=1$ & $n=2$ \\
\hline \hline$Q_{\alpha} Q_{\beta} Q_{\gamma} I_{(n)}^{\alpha \beta \gamma} / I_{(n)}$ & $q^{0} Q^{2}$ & $3 q^{0} Q^{4}$ & 0 \\
$Q_{\alpha} Q_{\beta} u_{\gamma} I_{(n)}^{\alpha \beta \gamma} / I_{(n)}$ & $Q^{2}-\frac{1}{2} q^{2}$ & $-3 q^{0} Q^{2}$ & $-6 Q^{4} L\left(q^{0}, q\right)$ \\
$Q_{\alpha} u_{\beta} u_{\gamma} I_{(n)}^{\alpha \beta \gamma} / I_{(n)}$ & $-q^{0}$ & $2 q^{2}-3 Q^{2}-2 Q^{2} L\left(q^{0}, q\right)$ & $12 q^{0} Q^{2} L\left(q^{0}, q\right)$ \\
$Q_{\alpha} \eta_{\beta \gamma} I_{(n)}^{\alpha \beta \gamma} / I_{(n)}$ & $q^{0}$ & $4 Q^{2}$ & 0 \\
$u_{\alpha} u_{\beta} u_{\gamma} I_{(n)}^{\alpha \beta \gamma} / I_{(n)}$ & $-1-\frac{3}{2} L\left(q^{0}, q\right)$ & $3 q^{0}+6 q^{0} L\left(q^{0}, q\right)$ & $2 q^{2}-24\left(q^{0}\right)^{2} L\left(q^{0}, q\right)$ \\
$u_{\alpha} \eta_{\beta \gamma} I_{(n)}^{\alpha \beta \gamma} / I_{(n)}$ & 1 & $-4 q^{0}$ & 0 \\
\hline
\end{tabular}

Table 2. Contractions of $I_{(n)}^{\alpha \beta \gamma}$ with the tensors in eq. (3.14) for $n=0,1,2$.

where the prefactors have been chosen for convenience. These relations can be inverted to give the coefficients in the expansion $I^{\mu \nu \rho}=\sum_{j} c_{j} T_{j}^{\mu \nu \rho}$, with the following results:

- The tensor expansion of $I^{\mu \nu \rho}$ at leading order has been given in eqs. (3.15) and (3.16).

- Tensor expansion of $I^{\mu \nu \rho}$ at $\mathcal{O}(Q)$

$$
\begin{aligned}
& c_{1}\left(q^{0}, q\right) / I_{(1)}=\frac{3}{q^{2}}+\frac{3\left(3 Q^{2}-2 q^{2}\right)}{q^{4}} L\left(q^{0}, q\right), \\
& c_{2}\left(q^{0}, q\right) / I_{(1)}=-\frac{2 q^{0}}{q^{2}}-\frac{6 q^{0} Q^{2}}{q^{4}} L\left(q^{0}, q\right), \\
& c_{3}\left(q^{0}, q\right) / I_{(1)}=-\frac{Q^{2}}{q^{2}}-\frac{3 q^{0} Q^{4}\left(5 Q^{2}-2 q^{2}\right)}{q^{6}} L\left(q^{0}, q\right), \\
& c_{4}\left(q^{0}, q\right) / I_{(1)}=-\frac{Q^{2}}{q^{2}} L\left(q^{0}, q\right), \\
& c_{5}\left(q^{0}, q\right) / I_{(1)}=0 \\
& c_{6}\left(q^{0}, q\right) / I_{(1)}=0 .
\end{aligned}
$$

- Tensor expansion of $I^{\mu \nu \rho}$ at $\mathcal{O}\left(Q^{2}\right)$

$$
\begin{aligned}
& A c_{1}\left(q^{0}, q\right) / I_{(2)}=\frac{q^{0}\left(5 Q^{2}-2 q^{2}\right)}{q^{4}}+\frac{3 q^{0}\left(5 Q^{4}-4 q^{2} Q^{2}+8 q^{4}\right)}{q^{6}} L\left(q^{0}, q\right), \\
& c_{2}\left(q^{0}, q\right) / I_{(2)}=\frac{Q^{2}\left(5 Q^{2}-4 q^{2}\right)}{q^{4}}+\frac{3 Q^{2}\left(5 Q^{4}-6 q^{2} Q^{2}+4 q^{4}\right)}{q^{6}} L\left(q^{0}, q\right), \\
& c_{3}\left(q^{0}, q\right) / I_{(2)}=-\frac{5 q^{0} Q^{4}}{q^{4}}-\frac{3 q^{0} Q^{4}\left(5 Q^{2}-2 q^{2}\right)}{q^{6}} L\left(q^{0}, q\right), \\
& c_{4}\left(q^{0}, q\right) / I_{(2)}=-\frac{q^{0} Q^{2}}{q^{2}}-\frac{3 q^{0} Q^{4}}{q^{4}} L\left(q^{0}, q\right), \\
& c_{5}\left(q^{0}, q\right) / I_{(2)}=-\frac{5 Q^{6}}{q^{4}}-\frac{3 Q^{6}\left(5 Q^{2}-2 q^{2}\right)}{q^{6}} L\left(q^{0}, q\right), \\
& c_{6}\left(q^{0}, q\right) / I_{(2)}=-\frac{Q^{4}}{q^{2}}-\frac{3 Q^{6}}{q^{4}} L\left(q^{0}, q\right) .
\end{aligned}
$$




\section{The integrals $I^{\mu \nu}$}

Table 3 gives the contractions of $I^{\mu \nu}$ with the following basis tensors

$$
\begin{aligned}
& T_{1}^{\mu \nu}=Q^{\mu} Q^{\nu}, \\
& T_{2}^{\mu \nu}=Q^{\mu} u^{\nu}+u^{\mu} Q^{\nu}, \\
& T_{3}^{\mu \nu}=u^{\mu} u^{\nu}, \\
& T_{4}^{\mu \nu}=\eta^{\mu \nu},
\end{aligned}
$$

with the constants $I_{(n)}$ given in eq. (B.1).

\begin{tabular}{r||c|c} 
& $n=0$ & $n=1$ \\
\hline \hline$Q_{\alpha} Q_{\beta} I_{(n)}^{\alpha \beta} / I_{(n+1)}$ & $-2 Q^{2}$ & 0 \\
$Q_{\alpha} u_{\beta} I_{(n)}^{\alpha \beta} / I_{(n+1)}$ & $2 q^{0}$ & $12 Q^{2}$ \\
$u_{\alpha} u_{\beta} I_{(n)}^{\alpha \beta} / I_{(n+1)}$ & $2+4 L\left(q^{0}, q\right)$ & $-24 q^{0} L\left(q^{0}, q\right)$ \\
$\eta_{\alpha \beta} I_{(n)}^{\alpha \beta} / I_{(n+1)}$ & -4 & 0 \\
\hline
\end{tabular}

Table 3. Contractions of $I_{(n)}^{\alpha \beta}$ with the tensors in eq. (C.1) for $n=0,1$.

The coefficients of the expansion $I^{\mu \nu}=\sum_{j} c_{j} T_{j}^{\mu \nu}$ are given by

- Tensor expansion of $I^{\mu \nu}$ at leading order

$$
\begin{aligned}
& c_{1}\left(q^{0}, q\right) / I_{(1)}=-\frac{2}{q^{2}}-\frac{6 Q^{2}-4 q^{2}}{q^{4}} L\left(q^{0}, q\right), \\
& c_{2}\left(q^{0}, q\right) / I_{(1)}=\frac{2 q^{0}}{q^{2}}+\frac{6 q^{0} Q^{2}}{q^{4}} L\left(q^{0}, q\right), \\
& c_{3}\left(q^{0}, q\right) / I_{(1)}=\frac{2 Q^{2}}{q^{2}}+\frac{6 Q^{4}}{q^{4}} L\left(q^{0}, q\right), \\
& c_{4}\left(q^{0}, q\right) / I_{(1)}=\frac{2 Q^{2}}{q^{2}} L\left(q^{0}, q\right) .
\end{aligned}
$$

- Tensor expansion of $I^{\mu \nu}$ at $\mathcal{O}(Q)$

$$
\begin{aligned}
& c_{1}\left(q^{0}, q\right) / I_{(2)}=-24 q^{0} L\left(q^{0}, q\right), \\
& c_{2}\left(q^{0}, q\right) / I_{(2)}=-12 Q^{2} L\left(q^{0}, q\right), \\
& c_{3}\left(q^{0}, q\right) / I_{(2)}=0, \\
& c_{4}\left(q^{0}, q\right) / I_{(2)}=0 .
\end{aligned}
$$

\section{References}

[1] D. T. Son and P. Surowka, Hydrodynamics with Triangle Anomalies, Phys.Rev.Lett. 103 (2009) 191601, [arXiv:0906.5044]. 
[2] Y. Neiman and Y. Oz, Relativistic Hydrodynamics with General Anomalous Charges, JHEP 1103 (2011) 023, [arXiv: 1011.5107].

[3] G. Newman, Anomalous hydrodynamics, JHEP 0601 (2006) 158, [hep-ph/0511236].

[4] D. E. Kharzeev and H. J. Warringa, Chiral Magnetic conductivity, Phys.Rev. D80 (2009) 034028, [arXiv:0907.5007].

[5] D. E. Kharzeev and D. T. Son, Testing the chiral magnetic and chiral vortical effects in heavy ion collisions, Phys.Rev.Lett. 106 (2011) 062301, [arXiv:1010.0038].

[6] K. Landsteiner, E. Megias, and F. Pena-Benitez, Gravitational Anomaly and Transport, Phys.Rev.Lett. 107 (2011) 021601, [arXiv:1103.5006].

[7] K. Jensen, M. Kaminski, P. Kovtun, R. Meyer, A. Ritz, et. al., Towards hydrodynamics without an entropy current, Phys.Rev.Lett. 109 (2012) 101601, [arXiv:1203.3556].

[8] K. Jensen, Triangle Anomalies, Thermodynamics, and Hydrodynamics, Phys.Rev. D85 (2012) 125017, [arXiv: 1203.3599].

[9] N. Banerjee, J. Bhattacharya, S. Bhattacharyya, S. Jain, S. Minwalla, et. al., Constraints on Fluid Dynamics from Equilibrium Partition Functions, JHEP 1209 (2012) 046, [arXiv: 1203.3544].

[10] M. Valle, Hydrodynamics in $1+1$ dimensions with gravitational anomalies, JHEP 1208 (2012) 113, [arXiv: 1206.1538].

[11] K. Jensen, R. Loganayagam, and A. Yarom, Thermodynamics, gravitational anomalies and cones, arXiv: 1207.5824 .

[12] W. A. Bardeen and B. Zumino, Consistent and Covariant Anomalies in Gauge and Gravitational Theories, Nucl.Phys. B244 (1984) 421. Revised version.

[13] A. Bilal, Lectures on Anomalies, arXiv:0802.0634.

[14] R. Loganayagam, Anomaly Induced Transport in Arbitrary Dimensions, arXiv:1106.0277.

[15] R. Loganayagam and P. Surowka, Anomaly/Transport in an Ideal Weyl gas, JHEP 1204 (2012) 097, [arXiv:1201.2812].

[16] L. Kadanoff and P. C. Martin, Hydrodynamic equations and correlation functions, Annals of Physics 24 (1963) 419-469.

[17] A. Rebhan, Collective phenomena and instabilities of perturbative quantum gravity at nonzero temperature, Nucl.Phys. B351 (1991) 706-734.

[18] R. C. Tolman and P. Ehrenfest, Temperature equilibrium in a static gravitational field, Physical Review 36 (1930) 1791.

[19] D. Z. Freedman and S.-Y. Pi, External Gravitational Interactions in Quantum Field Theory, Annals Phys. 91 (1975) 442.

[20] A. K. Rebhan and D. J. Schwarz, Kinetic versus thermal field theory approach to cosmological perturbations, Phys.Rev. D50 (1994) 2541-2559, [gr-qc/9403032].

[21] D. T. Son and N. Yamamoto, Berry Curvature, Triangle Anomalies, and Chiral Magnetic Effect in Fermi Liquids, arXiv: 1203.2697.

[22] M. Stephanov and Y. Yin, Chiral Kinetic Theory, arXiv:1207.0747. 
[23] D. T. Son and N. Yamamoto, Kinetic theory with Berry curvature from quantum field theories, arXiv: 1210.8158. 